\title{
HOT AIR TREATMENT, IN NATURAL CONVECTION CONDITIONS, FOR EGG SURFACE DECONTAMINATION
}

\author{
Chiara Cevoli, Angelo Fabbri, Frédérique Pasquali, Annachiara Berardinelli, Adriano Guarnieri
}

\section{Introduction}

Salmonella Enteritidis represents the most often serovar associated with human salmonellosis due to the consumption of contaminated eggs, and is the serovar causing more than $50 \%$ of human infections with Salmonella in Europe in 2006 [EFSA 2007].

By 2012, Council Directive 1999/74/EC, defining minimum standards for the welfare of laying hens, will abolish conventional cage systems in favour of enriched cages or floor systems in order to improve the welfare of hens. However, keeping hens on the floor or outside could present an increased risk to contamination as bacterial eggshell contamination with a total count of aerobic bacteria is generally significantly higher for nest eggs from non-cage systems compared to eggs from furnished or conventional cages [EFSA 2005].

In this regard the introduction of efficient measures to reduce eggshell contamination by bacterial pathogens, and thus to prevent any potential or additional food safety risk for human health, may be envisaged.

Even considering the potential problems due to treatment with water, hot air pasteurization may represent a valuable alternative for the decontamination of shell eggs. Since the hot air treatment of the eggs involves a simple and inexpensive plant, it can be evaluated, before packaging, as a promising industrial process. Nevertheless few studies have been per-

Paper received 03.05.2010; accepted 07.06.2010

Chiara Cevoli, PhD; Angelo Fabbri, researche assistant; AnNaCHIARA Berardinelli PhD; AdRiano GuARnieri, full professor - Department of Agricultural Economics and Engineering DEIAgra, University of Bologna, P.zza Goidanich, 60, Cesena FC. E-mail: afabbri @ foodsci.unibo.it

FrederiQue Pasquali PhD, Food Science Department DISA, University of Bologna, v.del Florio, 2, Ozzano Emilia BO.

Dr. Pasquali, carried on the microbial tests. Dr. Berardinelli contributed in quality tests. Prof Guarnieri was the responsible of the research. Dr. Fabbri and Cevoli made all the remaining research work.

The research was partially founded by MURST-PRIN founds. formed on the decontamination power of the hot air technique on table eggs and with little attention to quality preservation.

Pasteurization techniques based on hot air reported in literature may be classified in two categories substantially depending on the air speed value: forced and natural convection methods. As regards the latter the most documented experiences make use of convection ovens. Particularly Hou [1996] observed a 5 $\log _{10}$ reduction of the Salmonella Enteritidis load on yolk of eggs treated with a hot air oven at $55{ }^{\circ} \mathrm{C}$ for $180 \mathrm{~min}$. In addition, these researchers reported that heating internally contaminated shell eggs in water at $57^{\circ} \mathrm{C}$ for $25 \mathrm{~min}$, followed by their treatment with hot air at $55^{\circ} \mathrm{C}$ for $60 \mathrm{~min}$, resulted in microbial reductions by $7 \log _{10}$. Standelman [1996] and Hou [1996] reported that the Haugh unit, $\mathrm{pH}$, yolk index, and color showed no significant difference between fresh and pasteurized eggs in the oven, while albumen viscosity, turbidity and hue values showed significant differences that indicated partial protein denaturation.

As none of the documented research poses a specific attention to surface decontamination, the objective of this study was to develop and validate a numerical model useful to study the physics of treatment with hot air, in natural convection conditions. Then the impact of the treatment on egg quality and Salmonella Enteritidis inactivation on the shell was assessed.

\section{Materials and methods}

The tested treatments were characterised by an air temperature of $55^{\circ} \mathrm{C}$ for $50,100,150$ and 200 minutes. The thesis choice was driven by the experience of Hou [1996] of albumen coagulation limit conditions.

\subsection{CFD model development}

A numerical model was developed to describe the thermal interaction between the air and the egg, using a computational fluid dynamic tool (CFD) based on 
the Finite Element Technique (Comsol Multiphysics 3.5a, COMSOL Inc., Burlington, MA, USA).

The eggs are reasonably axisymmetric and consequently the 3D geometries can be generated by the revolution of the shell profile around the longitudinal axis. Moreover for the specific problem even the boundary condition was axisymmetric.

The Geometric profile of the egg was defined by the following equation [Narushin 1997] being $x$ and $y$ the cartesian coordinate in the plane:

$$
y= \pm 1.5396 \frac{B}{L} \sqrt{L^{05} x^{15}-x^{2}}
$$

The yolk were described as spherical $(r)$ while the shell thickness $(s)$ was considered uniform (Fig. 1). The parameters $L(0.058 \mathrm{~m}), B(0.044 \mathrm{~m}), r(0.015 \mathrm{~m}$, measured indirectly by volume measurement) and $s$ $(0.3 \mathrm{~mm})$ were determined through measurements carried out on 30 eggs from hens Hy-Line Brown using a digital calliper (CDJB15, Borletti, Italy).

The yolk, albumen and shell thermal properties were obtained experimentally by a needle probe KD2 (Decagon Device Inc., Pullman, USA) documented in a previous study [Fabbri 2007] and are reported in Table 1.

\subsection{Governing equation and boundary conditions}

The heat transfer is governed by the energy conservation equation, being the thermal diffusivity $D\left(\mathrm{~m}^{2} \mathrm{~s}^{-1}\right)$ different for every egg component:

$$
\frac{\partial T}{\partial t}=D\left(\frac{\partial^{2} T}{\partial x^{2}}+\frac{\partial^{2} T}{\partial y^{2}}+\frac{\partial^{2} T}{\partial z^{2}}\right)
$$

The initial temperature of the egg was considered uniform ( $\mathrm{T}=298 \mathrm{~K}$ ) while the boundary condition, imposed on the external surface of the shell, is purely convective:

$$
\dot{\mathrm{q}}=h\left(T_{\infty}-T\right)
$$

being $T_{\infty}$ the external bulk temperature $(328 \mathrm{~K})$.

The value of $h$ depends on the geometry and the ambient flow conditions. In this study it was obtained by the Nusselt number, being $k_{\text {air }}$ the air thermal conductivity:

$$
\mathrm{Nu}=\frac{h B}{k_{\text {air }}}
$$

In the case of natural convection, $\mathrm{Nu}$ depends on the Grashof number, Prandtl number, geometry and boundary conditions. Considering the egg like a sphere, the following equation, was considered [Raithby 2000]:

$$
\mathrm{Nu}-\left(\left(\mathrm{Nu}_{i}\right)^{\dagger}+\left(\mathrm{Nu}_{i}\right)^{\mathrm{i}}\right)^{\mathrm{L} \sigma}
$$

where:

$$
\begin{gathered}
\mathrm{Nu}_{\mathrm{f}}=2+0.878 \overline{C_{i}} \mathrm{Ra}^{14} \\
\mathrm{Nu},=\bar{C} \mathrm{Ra}^{1 / 3}
\end{gathered}
$$

$$
\begin{gathered}
\bar{C}_{\mathrm{r}}=0.671 /\left(1+(0.492 / \mathrm{Pr})^{9 / 16}\right)^{4 / 9} \\
\mathrm{Ra}=\mathrm{Gr} \cdot \mathrm{Pr} \\
\mathrm{Gr}=\frac{g \beta B^{3} \rho^{2}(\Delta T)}{\mu^{2}} \\
\operatorname{Pr}=\frac{c_{p} \mu}{k_{a x}} \\
g=9.8 \\
\beta=1 / \mathrm{T} \\
\bar{C}_{t}=0.11[\text { Raithby 2000]. }
\end{gathered}
$$

Furthermore, as reported on the Material Property Database (JAHM Software, Inc.) for air at normal pressure:

$$
\begin{aligned}
& C_{p}=-1.05 \mathrm{E}+03-3.73 \mathrm{E}-01 \mathrm{~T}+9.45 \mathrm{E}-04 \mathrm{~T}^{2}-6.02 \mathrm{E}-07 \mathrm{~T}^{3}+ \\
& 1.28 \mathrm{E}-10 \mathrm{~T}^{4} \\
& \mu=-75.20 \mathrm{E}-10 .+4.427 \mathrm{E}-8 T-7.887 \mathrm{E}-12 T^{2} \\
& k_{\text {air }}=-2.28 \mathrm{E}-03+1.155 \mathrm{E}-04 T-7.9 \mathrm{E}-08 T^{2}+4.12 \mathrm{E}-11 T^{3}- \\
& \text { 7.44E- } 15 T^{4} \\
& \rho=346.52 / \mathrm{T}
\end{aligned}
$$

The contact thermal resistance at the interface between shell and albumen and between albumen and yolk was neglected.

\subsection{Experimental validation of the CFD model}

To validate the numerical model, 10 eggs were treated in an oven (High performance oven, MOD 2100, F.lli Galli, Milan, Italy) at $55^{\circ} \mathrm{C}$ for a time of 50,100 , 150 and 200 minutes. During the tests, the temperature changes were measured on the internal surface of the shell and in the albumen (both in equatorial area) by two K-type thermocouples (Chromel/Alumel; Tersid Came; Italy). A $10 \mathrm{~mm}$ hole was drilled through the shell, then the yolk and albumen were extracted to insert and fix the internal thermocouple. Finally, the egg was refilled with only albumen.

The thermal data were sampled at a frequency of $250 \mathrm{~Hz}$, using the data acquisition system PCI-6036E and a virtual instrument created with LabView 8.2 (National Instrument Corporate; Texas,USA ).

The calculated time-temperature curves were than compared with the observed data.

\subsection{Quality tests}

Different quality traits were evaluated on eggs immediately after the treatment: turbidity of albumen, shell colour and the shell membranes assessment. 30 eggs were used as control (at time $\mathrm{t}=0$ ) and other $30 \times 4$ eggs were treated in an oven for 50, 100, 150 and 200 minutes.

The turbidity of albumen $\left(\tau_{a l b}\right)$ was used as an index of coagulation. Four millilitres of albumen were transferred to a translucent disposal PE cuvette (Sigma, Milan, Italy) and the transmission was measured 

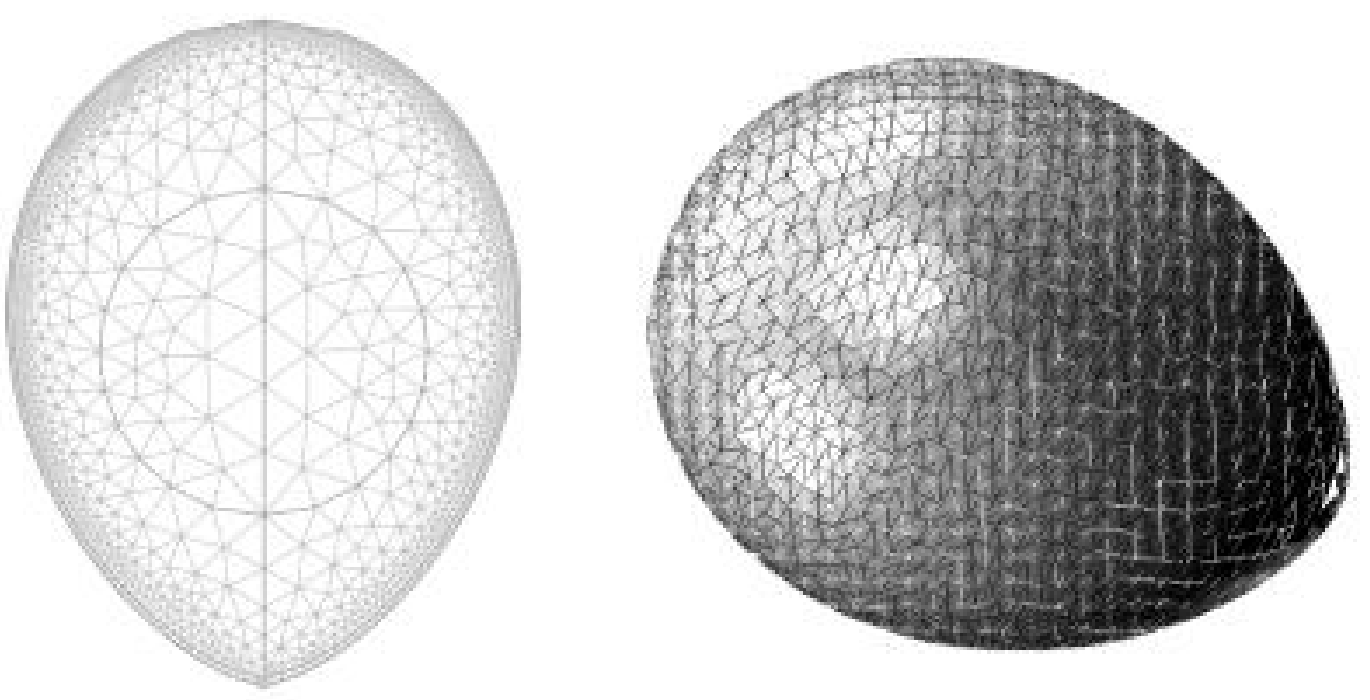

Fig. 1 - 3D geometry of eggshell, characterized by 35494 tetrahedral elements and 7031 nodes.

by a spectrophotometer at $600 \mathrm{~nm}$ (Spectrophotometer UV-1601, Shimadzu Corporation, Japan) as proposed by Pasquali [2010].

The exterior $L^{*}, a^{*}, b^{*}$ colour values of the shells, before and after the treatment, were measured with a CIELab colorimeter (Konica Minolta Meter CR-400, Milan, Italy). In the $L^{*} a^{*} b^{*}$ colour space, the difference was expressed as a single numerical value $\Delta E^{*}$. Three measurements were done on each egg in the same point and the value $\Delta E^{*}$ was calculated as follows [Francis 1975]:

$$
\Delta E^{*}=\sqrt{\left(\Delta L^{*}+\Delta a^{*}+\Delta b^{*}\right)}
$$

where $\Delta L^{*}, \Delta a^{*}, \Delta b^{*}$ were the differences of the average values measured before and after the treatment.

The shell membrane assessment was carried out indirectly evaluating the water loss of egg through the shell pores.

\subsection{Microbiological tests}

The contamination of eggs was assessed on $20 \times 4$ treated eggs (test), experimentally inoculated on the shell with Salmonella Enteritidis and on 10x4 inoculated untreated eggs (control).

The inoculate eggs were treated respectively for $50,100,150$ and 200 minutes at $55^{\circ} \mathrm{C}$ in the oven (High performance oven, MOD 2100, F.lli Galli, Milan, Italy)

The microbiological protocol (inoculum preparation, inoculation of the eggshell and quantification of eggshell) is described in detail by Pasquali [2010].

\section{Results and discussion}

In Figure 2 is showed the time-temperature curve calculated in three points inside the egg, respectively at the inner surface of the shell, in the albumen and in the yolk. It can be observed that the different lines are very close, especially after 100 minutes of treatment when the temperature values are stabilized at around $55^{\circ} \mathrm{C}$.

The simulation data were validated by experimental data obtained by thermocouples.

In Figure 3 the calculated and observed time-temperature curves, relative to a 200 minutes treatment at $55^{\circ} \mathrm{C}$ for one sample are compared. The figure is representative of the whole egg sample, for which the difference between calculated and measured data is always below $4.5^{\circ} \mathrm{C}$. This difference is probably due mainly to the simplification hypothesis made about testaceous membranes.

Figure 4 gives the mean values and standard deviations of albumen turbidity determined on treated and control eggs. The absence of significant differences between the two groups clearly shows that a treatment duration of 200 minutes does not activate any coagulation of the albumen.

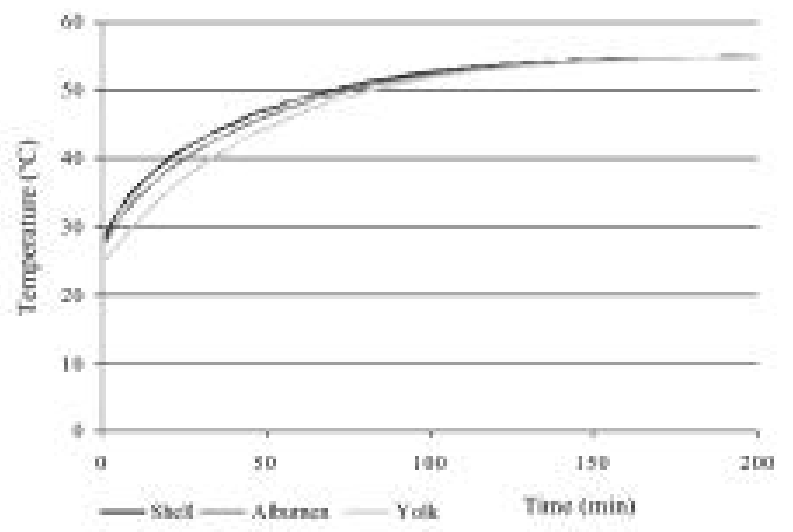

Fig. 2 - Time-temperature curves calculated in three points inside the egg (equatorial area: inner face of the shell, albumen and yolk) during a 200 minute treatment. 


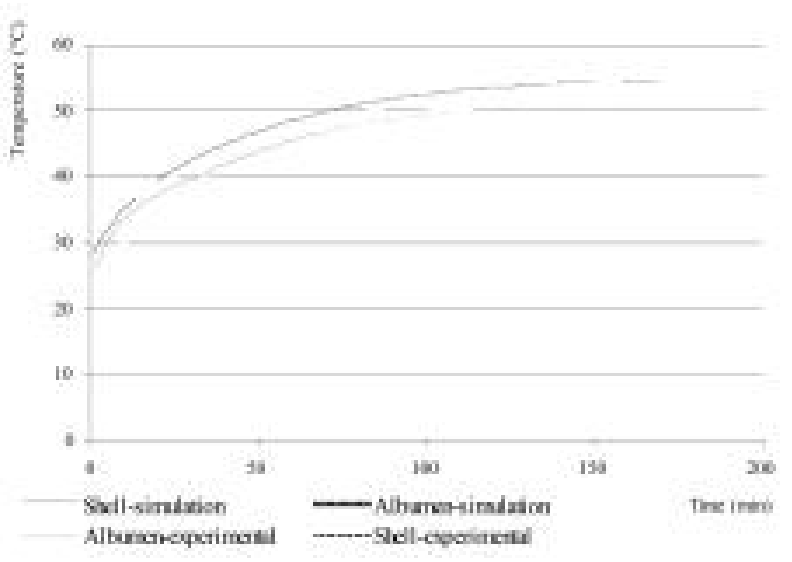

Fig. 3 - Observed time-temperature curves at the inner face of the shell and albumen, in equatorial area, during a 200 minute treatment.

In figure 5 the weight loss observed at 50, 100, 150 e 200 minutes of treatment is reported. It is possible to note that weight loss increases almost linearly but that the value reached at the end of the treatment $(0.285 \%)$ is very small and similar to the natural weight loss of eggs stored for the same time at environment temperature $(0.21 \%)$. It has to be recalled that the weight loss after deposition is a natural behaviour of eggs, due to water loss for evaporation. The damage of testaceous membranes can speed up the process.

In table 2 the mean values and standard deviations of $a^{*}, b^{*}, L^{*}$ parameters observed on treated and untreated eggs are reported. The ANOVA analysis does not report any significative difference between the two groups.

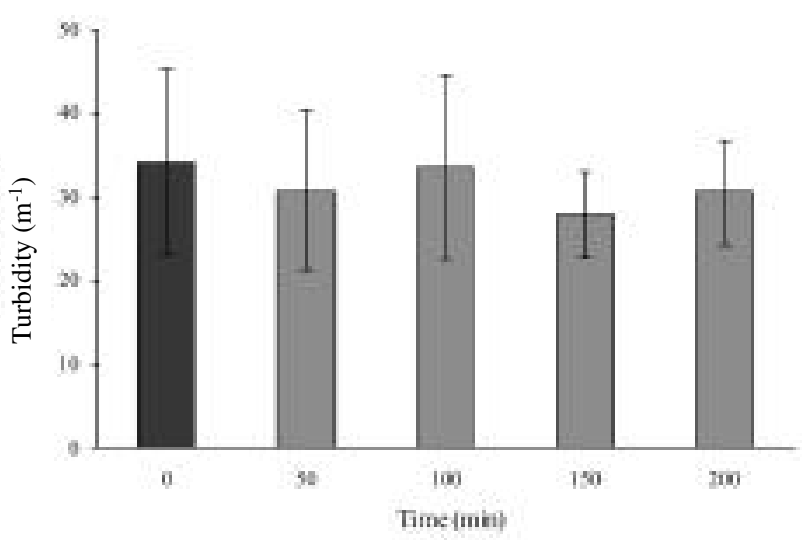

Fig. 4 - Turbidity values on treated and untreated samples of albumen. The control sample is represented in the darker colour.

Even the difference between $\Delta E^{*}$ values are not significant.

As regards the decontamination effectiveness in Figure 6 , the results of microbiological tests on eggs inoculated with Salmonella Enteritidis, treated and untreated are reported. It is possible to observe a reduction in microbial load up to $2.6 \log _{10}$ after 200 minutes. It is interesting to note that a linear correlation between the time of treatment and the microbial decimal reduction $\left(\mathrm{R}^{2} 0.92\right)$ exists, this suggests the opportunity of further study with a longer treatment. Moreover the results about decontamination effectiveness and weak action on quality parameters suggest the practical feasibility of a transportation system for eggs, from production to sorting plant, in heated containers.

\begin{tabular}{|c|c|c|c|}
\hline & Thermal conductivity $\left(\mathrm{Wm}^{-1} \mathrm{~K}^{-1}\right)$ & Specific heat $\left(\mathrm{Jkg}^{-1} \mathrm{~K}^{-1}\right)$ & $\left(\mathrm{kgm}^{-3}\right)$ \\
\hline Shell & 1.03 & 1234 & 2300 \\
\hline Abumen & 0.55 & 3860 & 1000 \\
\hline Yolk & 0.36 & 2849 & 1053 \\
\hline
\end{tabular}

TABLE 1 - Thermal properties of egg components.

\begin{tabular}{clcccc}
\hline \multicolumn{7}{c}{ Treatment } \\
\hline \multicolumn{7}{c}{$50 \mathrm{~min}$} & $100 \mathrm{~min}$ & $150 \mathrm{~min}$ & $200 \mathrm{~min}$ & Control \\
\hline$a^{*}$ & 16.452 .78 & 16.602 .86 & 16.302 .03 & 15.842 .05 & 16.471 .76 \\
$b^{*}$ & 30.021 .72 & 30.021 .54 & 30.031 .96 & 29.871 .46 & 30.121 .56 \\
$L^{*}$ & 62.852 .19 & 62.602 .14 & 62.981 .89 & 63.012 .56 & 62.801 .98 \\
$\Delta E^{*}$ & 0.65 & 0.66 & 0.63 & 0.69 & \\
\hline
\end{tabular}

TABLE 2 - Mean value and standard deviations of parameters $a^{*} b^{*} L^{*}$ treated and untreated samples. 


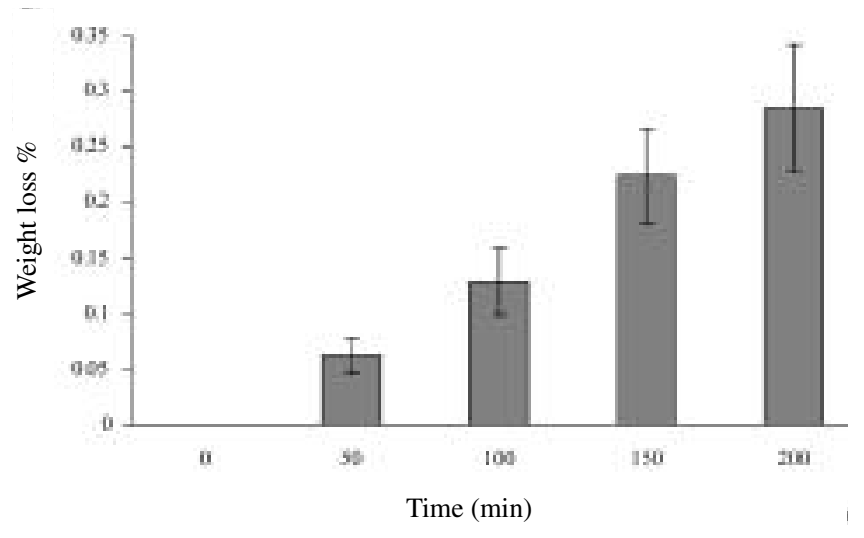

Fig. 5 - Weight loss observed on treated eggs.

\section{References}

European Food Safety Authority - The welfare aspects of various systems of keeping laying hens. The EFSA Journal, 2005,197, 1-23.

European Food Safety Authority - Report of the Task Force on Zoonoses Data Collection on the Analysis of the baseline study on the prevalence of Salmonella in holdings of laying hen flocks of Gallus gallus. The EFSA Journal, 2007, 97.

Fabbri A., Cevoli C., Sirri F., Measurement of thermal properties of table egg's components. In European Symposium on the quality Eggs and egg products. 2007, Prague, CZ.

Francis F.J., Clydesdale F.M., Food colorimetry: theory and applications., AVI Publishing, 1975, Westport, CT.

Hou H., Singh R.K., Muriana P.M., Stadelman W.J., Pasteurization of intact shell eggs. Food Microbiology, 1996, 13, 93-101.

Narushin V.G., The avian egg: geometrical description and calculation of parameters, Journal of Agricultural Engineering Research, 1997, 68, 201-205.

Pasquali F., Fabbri A., Cevoli C., Manfreda G., Franchini A., Hot Air Treatment for Surface Decontamination of Table Eggs. Food Control, 2010, 21, 431-435.

Raithby G.D., Terry K.G., Convection Heat Transfer CRC Handbook of Thermal Engineering. Ed. Frank Kreith,2000, Boca Raton: CRC Press LLC.

Stadelman W.J., Singh R.K., Muriana P.M., Hou H., Pasteurization of eggs in the shell. Poultry Science 1996, $75,1122-1125$.

\section{SUMMARY}

In order to improve the animal welfare, the Council Directive 1999/74/EC (defining minimum standards for the welfare of laying hens) will ban conventional cage systems from 2012, in favor of enriched cages or floor systems. As a consequence an increased risk of bacterial contamination of eggshells is expected. This has lead toward the development of efficient techniques to reduce eggshell contamination by bacterial pathogens, and thus to prevent any potential or

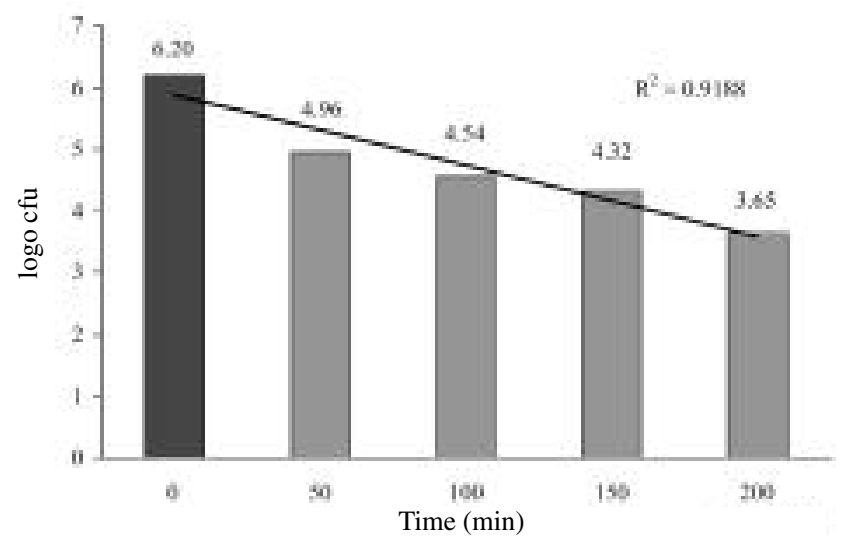

Fig. 6 - Microbial load of Salmonella Enteritidis ( $\log _{10}$ UFC) observed on shell of treated and control eggs.

additional food safety risk for Human health.

The objectives of this research were to develop and validate a numerical model useful to study the physics of treatment with hot air, in natural convection conditions, and then to assess the impact of the treatment on egg quality and Salmonella Enteritidis inactivation on the shell.

As regards the decontamination effectiveness, a reduction in microbial load up to $2.6 \log _{10}$ after 200 minutes was observed. A linear correlation between the time of treatment and the microbial decimal reduction $\left(\mathrm{R}^{2}\right.$ 0.92) was also observed. This suggests the opportunity of further study with longer treatment. Moreover the results about weak action on quality parameters suggest the practical feasibility of a transportation system for eggs, from production to sorting plant, in heated container.

Keywords: eggshell, hot air, CFD, thermal treatment, decontamination.

\section{Nomenclature}

$B$

$L \quad$ egg length (m) equatorial egg diameter $(\mathrm{m})$

$D \quad$ thermal diffusivity $\left(\mathrm{m}^{2} \mathrm{~s}^{-1}\right)$

$\dot{\mathrm{q}}$ heat flux $\left(\mathrm{W} \mathrm{m}^{-2}\right)$

$h \quad$ convective heat transfer coefficient $\left(\mathrm{W} \mathrm{m}^{-2} \mathrm{~K}^{-1}\right)$

$\mathrm{Nu} \quad$ Nusselt numbers

Gr Grashof number

$\mathrm{Pr} \quad$ Prandtl number

$\mathrm{Ra}$ Rayleigh numbers

$T$ temperature field (K)

$t \quad$ time (s)

$C_{p} \quad$ air specific heat $\left(\mathrm{J} \mathrm{K}^{-1} \mathrm{~kg}^{-1}\right)$

$\rho^{p} \quad$ air density $\left(\mathrm{kg} \mathrm{m}^{-3}\right)$

$g \quad$ acceleration gravity $\left(\mathrm{m} \mathrm{s}^{-2}\right)$

$\beta \quad$ air thermal expansion coefficient $\left(\mathrm{K}^{-1}\right)$

$k \quad$ thermal conductivity $\left(\mathrm{W} \mathrm{m}^{-1} \mathrm{~K}^{-1}\right)$

$\mu \quad$ air dynamic viscosity (Pa s) 
